\title{
Systematic review and meta-analysis of clinical efficacy of drug therapy for acute myelogenous leukemia
}

\author{
Huilin $\mathrm{Li}^{1 \#}$, Guanghong Wang ${ }^{2 \#}, \mathrm{Xia}^{\mathrm{W}} \mathrm{Wen}^{3,4}$, Lihua Zhou ${ }^{4,5}$ \\ ${ }^{1}$ Dermatology Outpatient Treatment Center, Institute of Dermatology and Venereal Diseases of Sichuan Provincial People's Hospital, Chengdu, \\ China; ${ }^{2}$ Oncology and Hematology, People's Hospital of the Pidu District, Chengdu, China; ${ }^{3}$ Neurosurgical Intensive Care Unit, Sichuan Provincial \\ People's Hospital, University of Electronic Science and Technology of China, Chengdu, China; ${ }^{4}$ Chinese Academy of Sciences Sichuan Translational \\ Medicine Research Hospital, Chengdu, China; ${ }^{5}$ Digestive Endoscopy Center, Sichuan Provincial People's Hospital, University of Electronic Science \\ and Technology of China, Chengdu, China \\ Contributions: (I) Conception and design: H Li, G Wang; (II) Administrative support: L Zhou; (III) Provision of study materials or patients: H Li, G \\ Wang, X Wen; (IV) Collection and assembly of data: All authors; (V) Data analysis and interpretation: L Zhou, G Wang, X Wen; (VI) Manuscript \\ writing: All authors; (VII) Final approval of manuscript: All authors. \\ "These authors contributed equally to this work. \\ Correspondence to: Lihua Zhou; Xia Wen. No. 32, West Section 2, Yihuan Road, Chengdu, China. Email: zlh0828342@163.com; z17790346420@163.com.
}

Background A meta-analysis was performed to examine the clinical efficacy of drugs in the treatment of acute myelogenous leukemia (AML).

Methods: combinations of terms of "acute myeloid leukemia", "fludara", "cytarabine", "cladribine", "aclacinomycin", "granulocyte colony-stimulating factor (G-CSF)", and "hyaluronic acid" were searched in Chinese databases. "acute myelogenous leukemia", "fludara", "cytarabine”, "cladribine”, "aclacinomycin", "granulocyte colony-stimulating factor", and "hyaluronic acid" were searched in English databases. Review Manager 5.3 was employed for the meta-analysis.

Results: Ultimately, 12 articles were included. Most of the articles had a low-risk bias, and were of medium or high quality. The complete remission rates were tested for heterogeneity [chi-square test $\left(\mathrm{Chi}^{2}\right)=4.10$, degrees of freedom $\left.(\mathrm{df})=10, \mathrm{I}^{2}=0 \% ; \mathrm{P}=0.94\right]$. The fixed effect model analysis showed that the difference between experimental participants and controls was considerable $[Z=13.15$, odds ratio $(\mathrm{OR})=12.82,95 \%$ confidence interval $(\mathrm{CI}):(8.77,18.76) ; \mathrm{P}<0.01]$. The overall effective rates were tested for heterogeneity $\left(\mathrm{Chi}^{2}=1.58, \mathrm{df}=7, \mathrm{I}^{2}=0 \% ; \mathrm{P}=0.98\right)$, and difference between experimental participants and controls was considerable $[\mathrm{Z}=10.70, \mathrm{OR}=1.32,95 \% \mathrm{CI}$ : $(7.32,17.89) ; \mathrm{P}<0.01]$. The overall adverse reaction rates were tested for heterogeneity $\left(\mathrm{Chi}^{2}=0.42, \mathrm{df}=5, \mathrm{I}^{2}=0 \% ; \mathrm{P}=0.99\right)$, and the difference between experimental participants and controls was considerable $[\mathrm{Z}=5.00, \mathrm{OR}=0.38,95 \% \mathrm{CI}:(0.26,0.55)$; $\mathrm{P}<0.01]$. The circles of some studies in the funnel diagrams were symmetrical with the midline, the accuracy of included trials was high, the publications were not biased, and the final conclusions were reliable.

Conclusions: This meta-analysis showed that drug treatments of AML can improve complete remission and total effective rates and reduce adverse reaction rates, and thus are worthy of clinical promotion.

Keywords: Drugs; acute myelogenous leukemia (AML); clinical efficacy

Submitted May 12, 2021. Accepted for publication Jun 18, 2021.

doi: 10.21037/apm-21-1390

View this article at: https://dx.doi.org/10.21037/apm-21-1390

\section{Introduction}

Acute myelogenous leukemia (AML) is a type of malignant blood disease that seriously endangers people's health. The incidence of AML increases with the increase of age, and the incidence of AML is about 5.06/100,000 in European countries and American (1). The incidence of leukemia, in 
European and American countries is about 5.06 per 100,000. In the past 20 years, the prognosis of AML patients younger than 65 years old has greatly improved, due to progress in diagnosis and prognosis stratification, the improvement of supportive treatment, and the finding of a solution to the worldwide problem for the lack of donor sources for allogeneic hematopoietic stem cell transplantation (2). The pathology of leukemia is mainly manifested by a decrease in the production of red blood cells, which leads to abnormal changes in the quality and quantity of white blood cells, and an abnormal increase in their number. It also causes infiltration into various organs and systems of the human body which damages the normal function of those organs. The main types of leukemia are acute lymphocytic, acute myeloid, chronic lymphocytic, and chronic myeloid; among which, chronic myeloid leukemia accounts for about $70 \%$ of all leukemias. Although standardized chemotherapy or hematopoietic stem cell transplantation can substantially improve the treatment effect of AML, the recurrence of most patients is still a difficult point in its treatment. Chemotherapy combined with immunotherapy is considered to be the most promising treatment, but the immune escape that easily occurs in the course of immunotherapy has become an important factor affecting the effect of leukemia treatment. Patients with leukemia can only be in complete remission before undergoing an autologous transplant. More and more evidence suggested that standardized diagnosis and prognostic stratification are the prerequisites and bases for selecting precise treatments and recurrence interventions for patients with AML (3).

In the 1980s, a randomized Eastern United States Oncology Cooperative Group study included maintenance drug therapy for the first time in a study of AML. This study compared patients who did not receive further medication to those who received long-term maintenance medication for 2 years. The results showed that the remission time of patients who did not receive maintenance medication was 4.1 months, which was substantially shorter than that of patients who received maintenance medication, and had a remission time of 8.1 months $(\mathrm{P}<0.05)$ (4). However, with these results, it was difficult to determine whether a drug-based treatment strategy was beneficial to the prognosis of patients with AML (5).

In recent years, the incidence of AML has gradually increased, and the curative effect has been poor (6). At present, common drug treatments for AML include fludara + cytarabine + granulocyte colony-stimulating factor (G-CSF) (FLAG), cladribine + cytarabine + G-CSF
(CLAG), aclacinomycin (ACR) and G-CSF (CAG), and hyaluronic acid + anthracycline (HAD) (7-9). Among these drug treatments, cytarabine combined with anthracycline therapy is the main treatment plan for AML. Currently, the physical fitness scores many elderly people gradually reduce due to the increase in the resistance rate to conventional chemotherapy and the mutation rate of drug-resistant genes, and they eventually die due to disease recurrence or complications (10). The efficacy of drug treatment programs remains highly controversial, and to date, no relevant systematic evaluation has been undertaken of these programs.

At present, drug therapy for acute myeloid leukemia is widely used in clinical practice, but the efficacy of drug therapy is still controversial, and there is no relevant systematic evaluation for the time being. What is innovative about this study is that after a large number of relevant literature on various drug therapy regimens were collected, meta-analysis was used to compare the clinical efficacy of drugs in the treatment of acute myeloid leukemia published since the establishment of the database with other therapeutic methods, to provide a theoretical basis for drug treatment of acute myeloid leukemia.

We present the following article in accordance with the PRISMA reporting checklist (available at https://dx.doi. org/10.21037/apm-21-1390).

\section{Methods}

\section{Article searching}

PubMed, Chinese Medical Citation Index (CMCI), Medline, Embase, China National Knowledge Network (CNKI), Wanfang, VIP and databases, and Google Scholar were searched to retrieve randomized controlled trials (RCTs) of drug treatments of AML published between the establishment of the databases and November 25, 2020. A compound Boolean logic was adopted to screen relevant articles. Chinese databases were searched by a combination of "acute myeloid leukemia", "fludara", "cytarabine", "cladribine", "aclacinomycin", "G-CSF", and "hyaluronic acid". English databases were searched by a combination of "acute myelogenous leukemia", "Fludara", "cytarabine", "cladribine", "aclacinomycin", "granulocyte colonystimulating factor", and "hyaluronic acid". According to the Rev Man 5.3 provided by the Cochrane system, the quality of each article was evaluated. The above search terms were combined freely, and a search engine was used to trace 
the search articles. Contact was made with professionals, researchers, and others in related field to ensure we had the latest research progress.

The above search terms were combined freely, and multiple searches were carried out to confirm inclusion in the literature. Search engines were used to track down certain documents. The title and abstract were screened first, and then the full text was evaluated. Professionals in relevant fields were contacted to keep abreast of research progress.

\section{Inclusion and exclusion criteria for articles}

Inclusion criteria: (I) reported on the results of RCT; (II) comprised patients who had been clinically diagnosed with AML; (III) conducted a pathological control analysis for which the index comparison was reliable in the $95 \% \mathrm{CI}$; and (IV) patients in experimental participants were treated with drugs for AML.

Exclusion criteria: (I) had overlapping subjects; (II) were about repeatedly published studies; (III) were about a relevant literature review or case report/s; (IV) incomplete data; and/or (V) the study examined a non-drug treatment for AML.

Two experts independently screened the title, abstract, and the full text, and 3 pre-experiments were required. If there was a disagreement between experts, it was discussed to reach a consensus or was arbitrated by a third party.

\section{Data extraction}

Two experts independently used a unified Excel sheet to list the data. If there was a disagreement, a consensus was reached by them or arbitrated by a third one. The extracted data included: (I) title; (II) name of the first author and year of publication; (III) publication name; (IV) date of the publication of research; (V) general information about subjects (i.e., average age, gender, and sample size); (VI) intervention measures for experimental and control groups; (VII) extracted evaluation indexes (i.e., the complete response rate, total effective rate, recurrence rate within 6 months, and the incidence of adverse reactions).

\section{Risk of bias evaluation}

Cochrane Collaboration tool was employed for the "risk of bias assessment" of the RCTs. The evaluation regarded the generation of a random assignment scheme, blind selection, hiding of an assignment scheme, integrity of data results, and results. These five aspects were categorized as having high, low, or unclear risk bias".

\section{Quality assessment}

The article quality was assessed by two researchers. If there was a disagreement between experts, it was discussed to reach a consensus or was arbitrated by a third party. The grading of recommendations, assessment, development and evaluation (GRADE) standard of Cochrane Collaboration was adopted for quality grading. The selected articles that received a score of 6 or above were deemed as high quality (i.e., as having a low risk of bias), those that received a score of 1 or less were deemed as low quality (i.e., as having a high risk of bias), and those that received a score of $2-5$ were considered medium quality (i.e., as having an unclear risk of bias).

\section{Statistical methods}

StataSE 12.0 was employed. Odds ratios (ORs) and 95\% CIs were calculated to reflect and compare the complete remission rates, total effective rates, recurrence rates within 6 months, and adverse reaction rates of patients with AML. The risk of bias assessment chart of Review Manager 5.3 was utilized to assess the included articles. Each effect was represented by a $95 \%$ CI. When $\mathrm{P}>0.01$ and $\mathrm{I}^{2}<50 \%$, a fixed-effects model (FEM) was adopted for the metaanalysis. When $\mathrm{P}<0.01$ and $\mathrm{I}^{2}>50 \%$, a random-effects model (REM) was employed.

\section{Results}

\section{Search results}

A total of 549 literatures were obtained in this study. Among them, 138 were eliminated because of abstracts and titles, 75 were eliminated because of repeated research subjects, and 31 were eliminated because of full text. Twelve articles were finally included in meta-analysis. Figure 1 shows a flow chart of the search process. Figure 2 shows the GRADE quality classification results. Seven articles received a score of 6 and above, 4 received a score of $2-5$, and 1 received a score of 1 or less.

Eleven articles, comprising 986 patient cases, met the inclusion criteria. Each of the 12 articles had a small sample size (the sample sizes ranged from 25 to 59 cases), and 


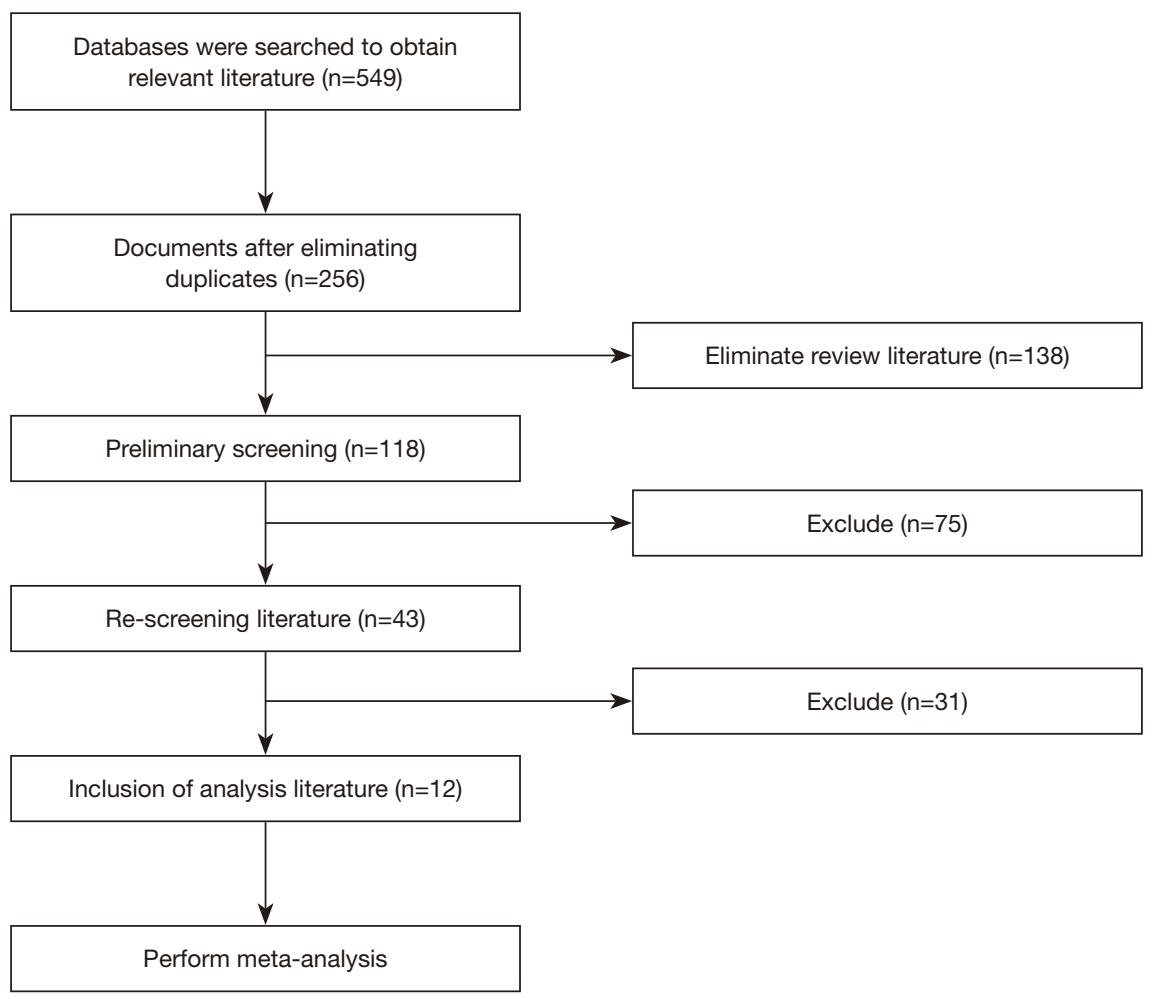

Figure 1 Article retrieval flow chart.

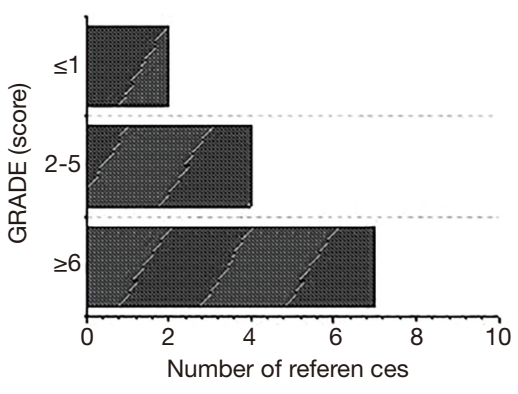

Figure 2 GRADE quality classification results.

all the research subjects were aged over 18 . The number of cases, the intervention measures, and other details of experimental participants and controls were described in detail in the 12 articles. Information about the research subjects is set out in Table 1 .

\section{Risk-bias evaluation of articles}

Figures 3 and 4 were multiple risk bias evaluation of the included articles. Among the 12 RCTs in this study, 4 RCTs (11-14) described the correct random allocation. Only
2 articles $(15,16)$ described the correct random allocation and concealment of the allocation plan. One article (17) was evaluated by blinding, while the other articles did not. However, the indicators were laboratory indicators determined by computer; thus, all articles were considered to have been blinded correctly.

\section{Meta-analysis results of complete remission rate}

Ten articles analyzed the complete remission rate of randomized controlled trials. There were 888 cases in total, 447 were experimental participants and 441 were controls. The overall heterogeneity test showed that chi-square test $\left(\mathrm{Chi}^{2}\right)=4.10$, degrees of freedom $(\mathrm{df})=10, \mathrm{I}^{2}=0 \%, \mathrm{P}=0.94$. The research of Ito $\mathrm{N}$ [2019] occupied the highest percentage (15.2\%) in the final combined results, followed by Liesveld JL [2015] (13.8\%). Horizontal line (HL) of the $95 \%$ confidence interval of all the studies crossed invalid vertical line (IVL). The FEM was adopted, and it was found that the complete remission rate of experimental participants was considerable compared with controls, $\mathrm{Z}=13.15, \mathrm{OR}=12.82,95 \% \mathrm{CI}$ : $(8.77$, 18.76), $\mathrm{P}<0.01$ (Figure 5).

Figure 6 was a funnel plot of the complete remission rate. 
Table 1 Information of the research objects in the articles

\begin{tabular}{|c|c|c|c|c|c|}
\hline Author & Year & \multicolumn{2}{|r|}{ Experimental group } & \multicolumn{2}{|c|}{ Control group } \\
\hline Cheson BD & 2003 & 26 & Fludara 20 mg, cytarabine $300 \mu \mathrm{g} / \mathrm{d}$ & 14 & Targeted therapy \\
\hline Ito $\mathrm{N}$ & 2019 & 29 & Aclacinomycin $20 \mathrm{mg}$, cytarabine $300 \mu \mathrm{g} / \mathrm{d}$ & 19 & Targeted therapy \\
\hline Lichtman MA & 2005 & 38 & Aclacinomycin 20 mgcytarabine $300 \mu \mathrm{g} / \mathrm{d}$, & 8 & Immunity therapy \\
\hline Reikvam H & 2014 & 48 & Cladribine $20 \mathrm{mg}$, cytarabine $300 \mu \mathrm{g} / \mathrm{d}$ & 21 & Targeted therapy \\
\hline Bruserud $\mathrm{O}$ & 2000 & 57 & Aclacinomycin $20 \mathrm{mg}$, cytarabine $300 \mu \mathrm{g} / \mathrm{d}$ & 29 & Immunity therapy \\
\hline Liesveld JL & 2005 & 59 & 54.8 & 28 & Targeted therapy \\
\hline Lorsbach RB & 1999 & 36 & Aclacinomycin $20 \mathrm{mg}$, cytarabine $300 \mu \mathrm{g} / \mathrm{d}$ & 19 & Targeted therapy \\
\hline Vasvani S & 2020 & 28 & Aclacinomycin $20 \mathrm{mg}$, cytarabine $300 \mu \mathrm{g} / \mathrm{d}$ & 6 & Immunity therapy \\
\hline Goa KL & 1994 & 59 & Aclacinomycin $20 \mathrm{mg}$, cytarabine $300 \mu \mathrm{g} / \mathrm{d}$ & 27 & Targeted therapy \\
\hline
\end{tabular}

Attention should be paid to the number of white blood cells during the use of cytarabine, which should be stopped when white blood cells is greater than $20 \times 10^{9} / \mathrm{L}$.

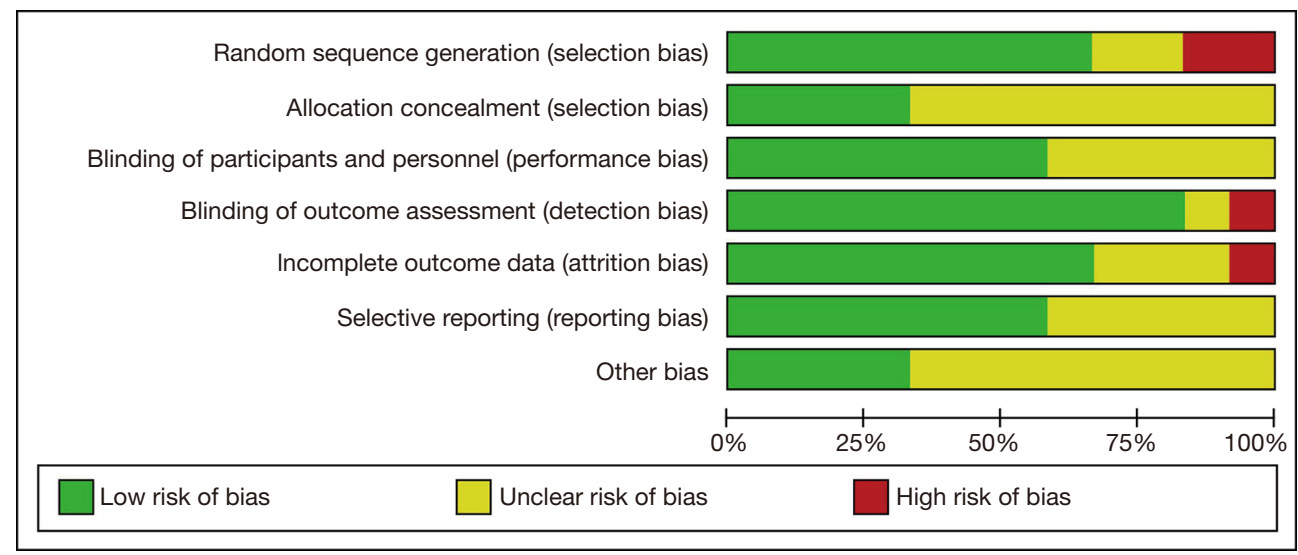

Figure 3 Risk bias evaluation of literatures.

The circles of the 11 studies were mainly distributed near the midline and were basically symmetrical, indicating that accuracy of those trials was high, the publication was not biased, and the final conclusions were reliable.

\section{Meta-analysis results for total effective rates}

Eight articles analyzed the total effective rates of the RCTs. There were 642 patients in total (comprising 323 patients in experimental participants and 319 patients in controls). An overall heterogeneity test was performed $\left(\mathrm{Chi}^{2}=1.58, \mathrm{df}=7\right.$, $\left.\mathrm{I}^{2}=0 \% ; \mathrm{P}=0.98\right)$. The results of Reikvam [2014] occupied the highest percentage $(19.8 \%)$ of the final combined results, with those of Goa ranked the second [1994] (17.4\%). HL of the $95 \%$ CIs of the 8 articles was to the right of IVL. A FEM was adopted for the meta-analysis, and difference between experimental participants and controls was substantial $[Z=10.70, \mathrm{OR}=1.32,95 \% \mathrm{CI}$ : $(7.32,17.89)$; $\mathrm{P}<0.01$; see Figure 7].

Figure 8 was funnel plot of the total effective rate. 


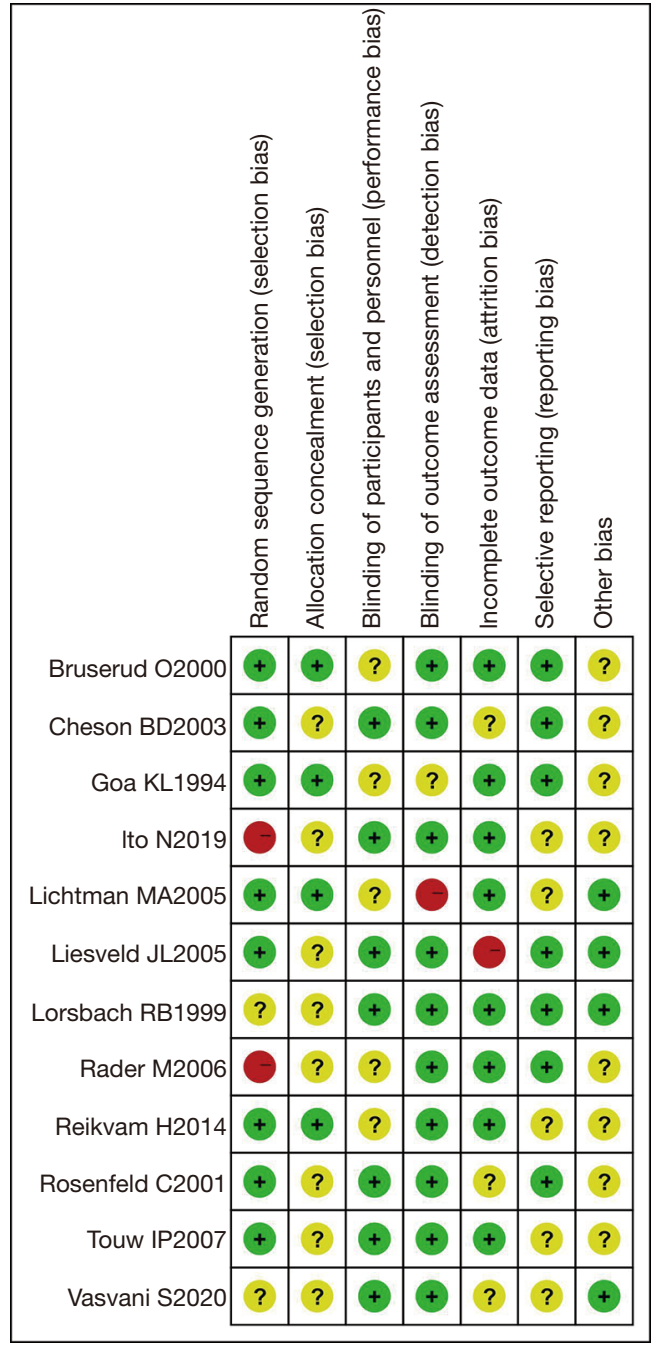

Figure 4 Multiple risk bias evaluation results. +, -, and ? mean low, high, and unclear risk bias, respectively.
The circles of the 8 studies were mainly distributed near and were basically symmetrical with midline as the axis, indicating that the accuracy of those trials was high, and no bias in publication was indicated.

\section{Meta-analysis results for recurrence rates within 6 months}

5 articles analyzed the recurrence rates within 6 months in the RCTs. There were 415 patients in total (comprising 208 patients in experimental participants and 207 patients in controls). An overall heterogeneity test was performed $\left(\mathrm{Chi}^{2}=1.05, \mathrm{df}=4, \mathrm{I}^{2}=0 \% ; \mathrm{P}=0.90\right)$. Lorsbach's [1999] results occupied the highest percentage of the final combined results (24.2\%), with Rosenfeld's as the second [2001] (23.5\%). HL of the $95 \%$ CI of the 2 articles crossed the left side of IVL. A FEMs was utilized, and the results showed that experimental participants differed substantially from controls $[\mathrm{Z}=4.74, \mathrm{OR}=0.31,95 \% \mathrm{CI}$ : $(0.19,0.50)$; $\mathrm{P}<0.01$; Figure 9].

Figure 10 was a funnel plot illustrating recurrence rate within half a year. The circles of the five studies were symmetrical with the midline as the axis, indicating no bias in publication.

\section{Meta-analysis results for adverse reaction rates}

Six articles analyzed the adverse reaction rates in the RCTs. There were 457 patients in total (comprising 229 patients in experimental participants and 228 patients in controls). In experimental participants, there were 21 cases of oral ulcers, 15 cases of nausea and vomiting, 46 cases of infection, and 18 cases of elevated serum transaminase. In controls, there

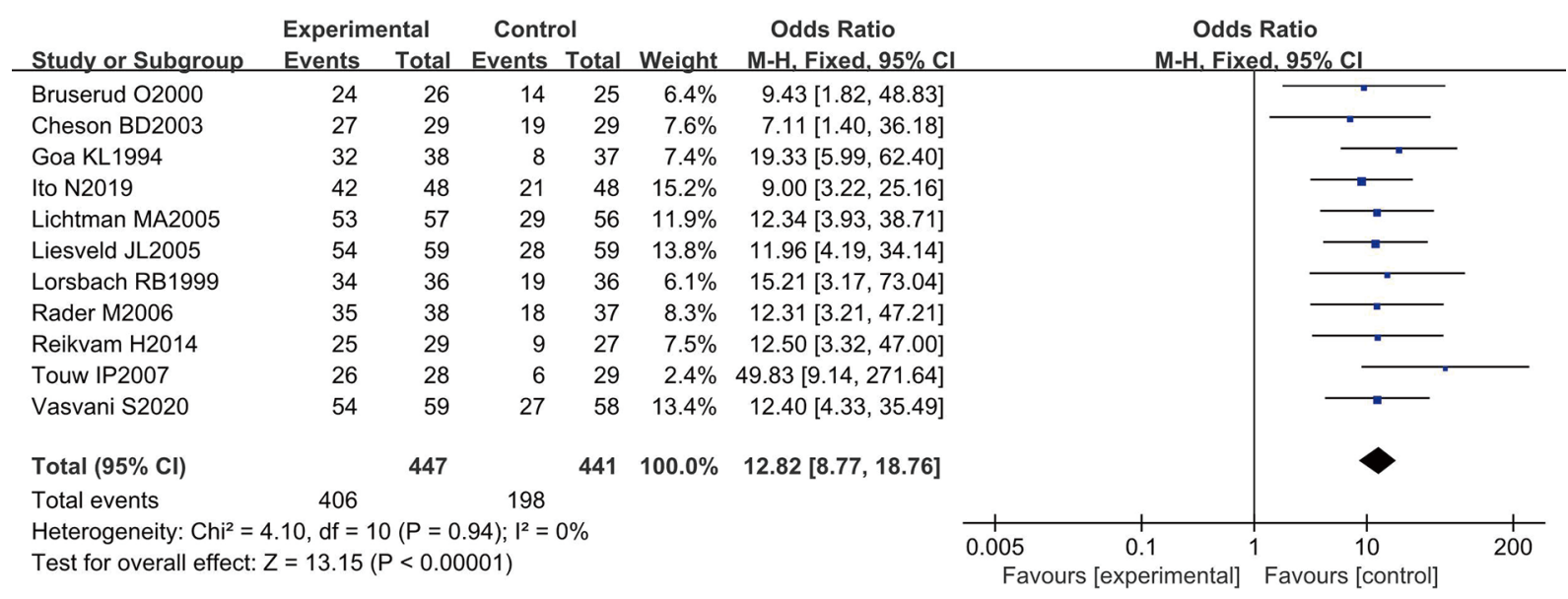

Figure 5 Forest plot of complete remission rates under a FEM. Chi ${ }^{2}$ means chi-square test, and df represents the degree of freedom. 


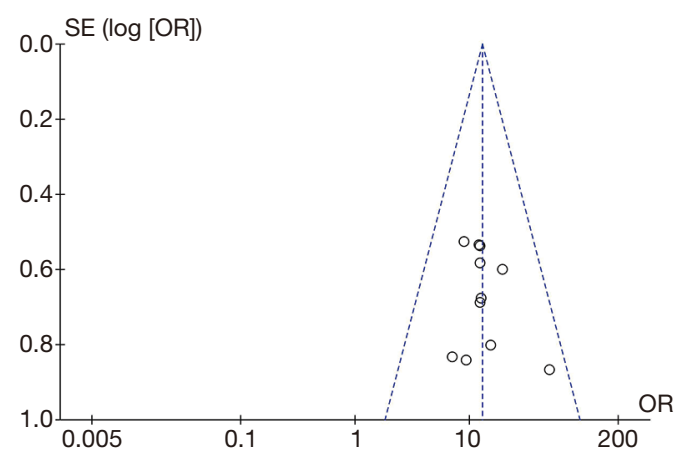

Figure 6 Funnel plot of complete remission rates.

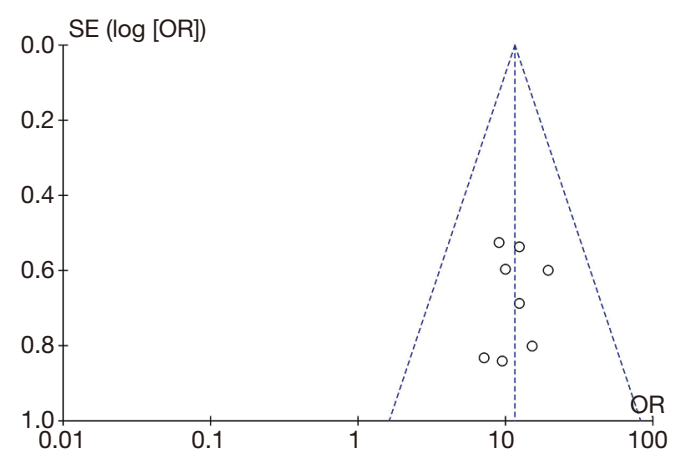

Figure 8 Funnel plot of the total effective rates.

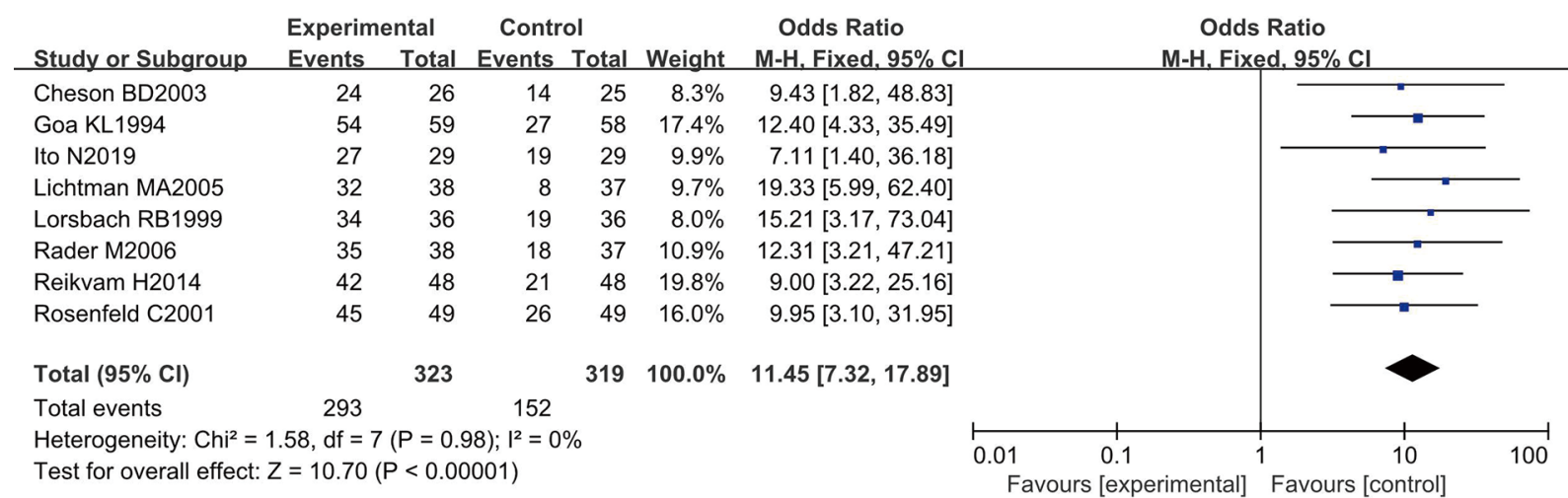

Figure 7 Forest plot of the total effective rates under a FEM. Chi ${ }^{2}$ means chi-square test, and df represents the degree of freedom.

were 46 cases of oral ulcers, 41 cases of nausea and vomiting, 52 cases of infection, and 53 cases of elevated serum transaminase. An overall heterogeneity test was performed $\left(\mathrm{Chi}^{2}=0.42, \mathrm{df}=5, \mathrm{I}^{2}=0 \% ; \mathrm{P}=0.99\right)$. Liesveld's [2005] results occupied the highest percentage of the final combined results (25.7\%), followed by those of Rosenfeld [2001] (22.7\%). HL of the $95 \%$ CIs of 3 articles crossed the left side of IVL. FEM analysis revealed that difference between controls and experimental participants was substantial $[\mathrm{Z}=5.00, \mathrm{OR}=0.38$, 95\% CI: $(0.26,0.55) ; \mathrm{P}<0.01$; see Figure 11].

Figure 12 was a funnel plot of the adverse reaction rate. The circles of the 6 studies were mainly concentrated near the midline, indicating no bias in publication.

\section{Discussion}

AML is the most common acute leukemia in adults. It is also a group of hematologic malignancies with high heterogeneity. It is characterized by clonal proliferation and infiltration of a large number of abnormal differentiated myeloid progenitor cells in bone marrow, blood or tissue. Moreover, it has high degree of malignancy and poor prognosis. Cytarabine (Ara-C) combined with anthracycline chemotherapy is the most important treatment for AML at present. After induction therapy, $20-40 \%$ of patients still can't achieve complete remission, and most patients will develop primary or acquired resistance. To find an effective treatment and medicine for AML, a meta-analysis was performed to compare the clinical efficacy of drugs in the treatment of AML to other non-drug treatments, such as immunotherapy and targeted therapy. The results showed that the accuracy of the included studies was high, there was no bias in the publications, and the final conclusions were credible (18).

The complete remission rates of the RCTs were analyzed and the overall heterogeneity was tested $\left(\mathrm{Chi}^{2}=4.10, \mathrm{df}=10\right.$, $\left.\mathrm{I}^{2}=0 \% ; \mathrm{P}=0.94\right)$. HL of the $95 \% \mathrm{CI}$ of the 10 studies crossed IVL. The experimental participants were considerable different compared to controls $[Z=13.15$, $O R=12.82$, $95 \%$ CI: $(8.77,18.76) ; \mathrm{P}<0.01]$. Thus, the results showed 


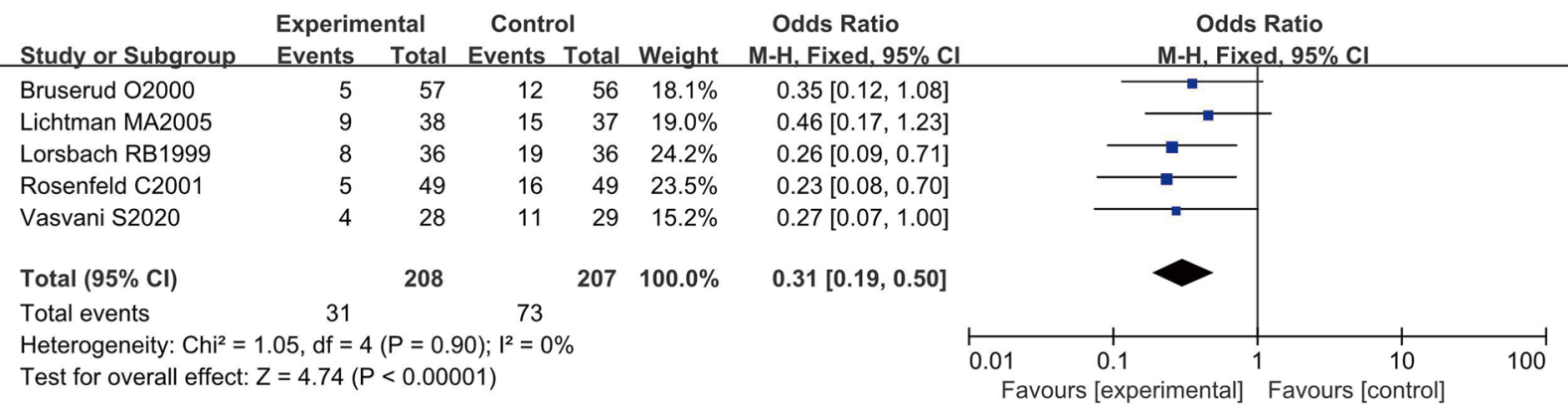

Figure 9 Forest plot of the recurrence rates within 6 months under a FEM. Chi ${ }^{2}$ means chi-square test, and df represents the degree of freedom.

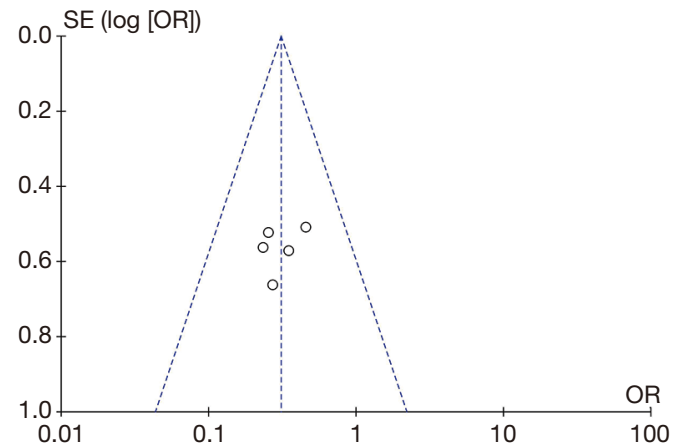

Figure 10 Funnel plot of the recurrence rates within 6 months.

that patients who received drug treatments had a higher complete remission rate than those who received other treatments. Additionally, drug treatments can substantially alleviate the pain of patients, and have obvious advantages.

The total effective rates in the RCTs were analyzed, and the overall heterogeneity was tested $\left(\mathrm{Chi}^{2}=1.58, \mathrm{df}=7, \mathrm{I}^{2}=0 \%\right.$, $\mathrm{P}=0.98)$. HL of the $95 \% \mathrm{CI}$ of the 8 articles was to the right of IVL. The difference between experimental participants and controls was substantial $[\mathrm{Z}=10.70, \mathrm{OR}=1.32,95 \% \mathrm{CI}$ : (7.32, 17.89); $\mathrm{P}<0.01]$. Thus, drug treatment appears to substantially inhibit the proliferation of cancer cells and promote the overall apoptosis of cancerous lesions.

The adverse reaction rates in the RCTs were analyzed. In experimental participants, there were 21 cases of oral ulcers, 15 cases of nausea and vomiting, 46 cases of infection, and 18 cases of elevated serum transaminase. In controls, there were 46 cases of oral ulcers, 41 cases of nausea and vomiting, 52 cases of infection, and 53 cases of elevated serum transaminase. An overall heterogeneity test was performed $\left(\mathrm{Chi}^{2}=0.42, \mathrm{df}=5, \mathrm{I}^{2}=0 \% ; \mathrm{P}=0.99\right)$. FEM was employed, and difference between controls and experimental participants was considerable $[\mathrm{Z}=5.00$, $\mathrm{OR}=0.38,95 \% \mathrm{CI}:(0.26,0.55) ; \mathrm{P}<0.01]$. Thus, drug treatments reduce the adverse reactions, such as lung infection, nausea, and vomiting. When medications are administered, preventive work should be undertaken as soon as possible, and practitioners should be alert to fever and lung infections. During treatment, the actual condition of a patient's body should be fully considered. If the pain becomes intolerable, the medication regimen should be adjusted immediately or the treatment should be stopped. The use of fungi and antibacterial drugs should be properly adjusted to reduce the treatment and prognosis of adverse reactions among patients and improve clinical efficacy.

Meta-analysis was implemented to systematically evaluate the clinical efficacy and safety of drugs for AML, and we found a complete remission rate of up to $94.4 \%$. Consistent with the results of this meta-analysis, Myles et al. [2017] (19) used drugs to treat AML and found a complete remission rate of $85.7 \%$. In this study, different methods were used to treat patients, and the dosage and types of drugs were not exactly the same, which may have affected patients' prognoses (20). In the future, a multi-center and large-sample RCT is required to evaluate the efficacy of the methods.

Of the 12 RCTs examined, only 2 described the correct randomized allocation and provided detailed descriptions of the concealment of the allocation plans. All the articles used a correct blinding method. The results of the GRADE quality classification showed that 7 articles received a score of 6 and above. The sample size of the research included in the meta-analysis of this study was small; however, the quality of the research was medium or high, and the quality level of the included experiments was reasonable. 


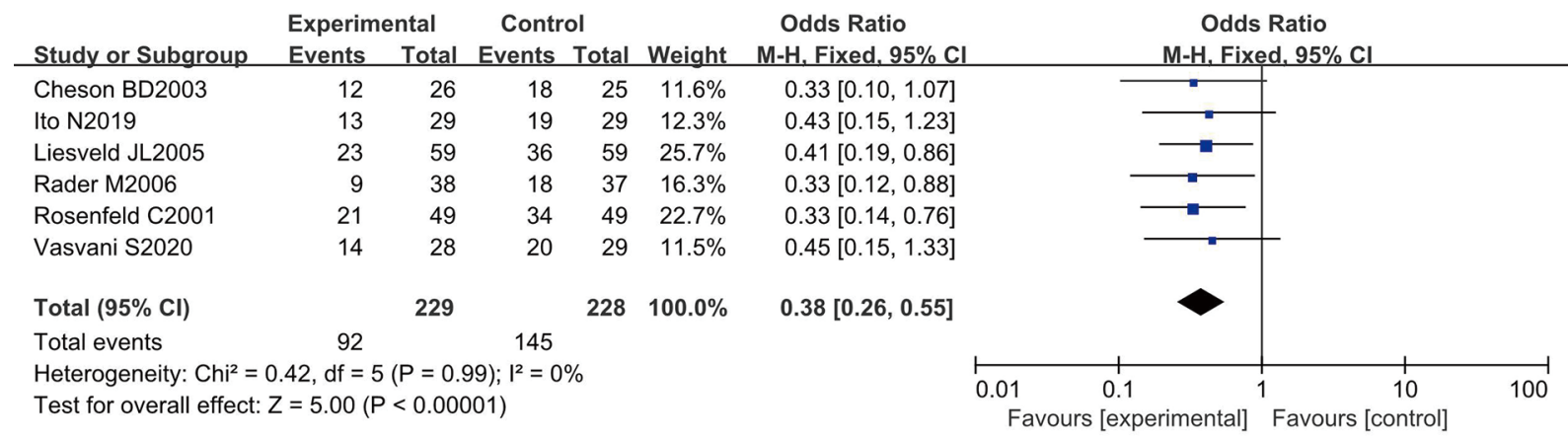

Figure 11 Forest plot of adverse reaction rates under a FEM. Chi ${ }^{2}$ means chi-square test, and df represents the degree of freedom.

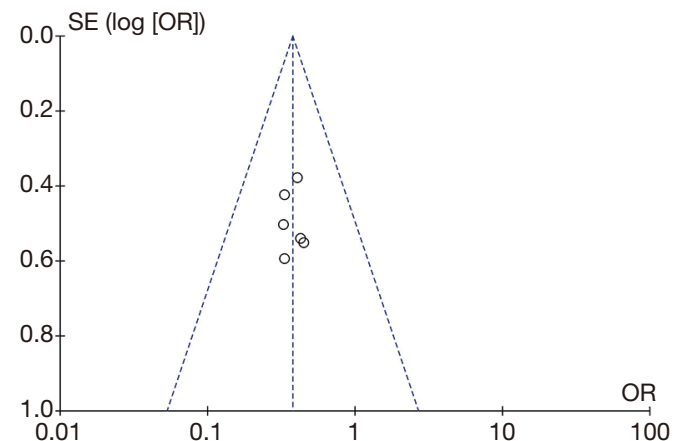

Figure 12 Funnel plot of adverse reaction rates.

\section{Conclusions}

After 12 relevant articles were analyzed to explore the clinical efficacy of drugs in the treatment of AML, it was found that experimental participants comprised patients who received drug treatments for AML and controls comprised patients who received other non-drug treatment methods. In addition, the use of drugs in AML improved the complete remission and total effective rates, and reduced the adverse reaction rate. Thus, drug treatments are worthy of clinical promotion. The limitations of this study lie in the low quality of the research objects, high heterogeneity, and small sample sizes. Thus, sample size will be expanded to conduct clinical RCTs to verify these results. In conclusion, this study, which performs Meta-analysis on drug treatment of AML, provides scientific evidence-based basis for clinical practice.

\section{Acknowledgments}

Funding: None.

\section{Footnote}

Reporting Checklist: The authors have completed the PRISMA reporting checklist. Available at https://dx.doi. org/10.21037/apm-21-1390

Conflicts of Interest: All authors have completed the ICMJE uniform disclosure form (available at https://dx.doi. org/10.21037/apm-21-1390). The authors have no conflicts of interest to declare.

Etbical Statement: The authors are accountable for all aspects of the work in ensuring that questions related to the accuracy or integrity of any part of the work are appropriately investigated and resolved.

Open Access Statement: This is an Open Access article distributed in accordance with the Creative Commons Attribution-NonCommercial-NoDerivs 4.0 International License (CC BY-NC-ND 4.0), which permits the noncommercial replication and distribution of the article with the strict proviso that no changes or edits are made and the original work is properly cited (including links to both the formal publication through the relevant DOI and the license). See: https://creativecommons.org/licenses/by-nc-nd/4.0/.

\section{References}

1. Cheson BD, Bennett JM, Kopecky KJ, et al. Revised recommendations of the International Working Group for Diagnosis, Standardization of Response Criteria, Treatment Outcomes, and Reporting Standards for Therapeutic Trials in Acute Myeloid Leukemia. J Clin Oncol 2003;21:4642-9. 
2. Ito N, Yoshida T, Ichikawa R, et al. Clinical and pathological characteristics of acute myelogenous leukemia in a female koala with diabetes mellitus. J Vet Med Sci 2019;81:1229-33.

3. Lichtman MA, Segel GB. Uncommon phenotypes of acute myelogenous leukemia: basophilic, mast cell, eosinophilic, and myeloid dendritic cell subtypes: a review. Blood Cells Mol Dis 2005;35:370-83.

4. Rosenfeld C, Kantarjian H. Is myelodysplastic related acute myelogenous leukemia a distinct entity from de novo acute myelogenous leukemia? Potential for targeted therapies. Leuk Lymphoma 2001;41:493-500.

5. Reikvam H, Brenner AK, Nepstad I, et al. Heat shock protein 70 - the next chaperone to target in the treatment of human acute myelogenous leukemia? Expert Opin Ther Targets 2014;18:929-44.

6. Bruserud O, Gjertsen BT. New strategies for the treatment of acute myelogenous leukemia: differentiation induction--present use and future possibilities. Stem Cells 2000;18:157-65.

7. Liesveld JL, Rosell KE, Lu C, et al. Acute myelogenous leukemia--microenvironment interactions: role of endothelial cells and proteasome inhibition. Hematology 2005;10:483-94.

8. Lorsbach RB, Folkerth RD, Pinkus GS. Relapse of acute myelogenous leukemia as a cerebellar myeloblastoma showing megakaryoblastic differentiation. Mod Pathol 1999;12:1186-91.

9. Rader M. Granulocyte colony-stimulating factor use in patients with chemotherapy-induced neutropenia: clinical and economic benefits. Oncology (Williston Park) 2006;20:16-21.

10. Touw IP, van de Geijn GJ. Granulocyte colony-stimulating factor and its receptor in normal myeloid cell development, leukemia and related blood cell disorders. Front Biosci 2007;12:800-15.

11. Vasvani S, Kulkarni P, Rawtani D. Hyaluronic acid: A review on its biology, aspects of drug delivery, route of administrations and a special emphasis on its approved marketed products and recent clinical studies. Int J Biol Macromol 2020;151:1012-29.

12. Goa KL, Benfield P. Hyaluronic acid. A review of its

Cite this article as: Li $\mathrm{H}$, Wang $\mathrm{G}$, Wen $\mathrm{X}$, Zhou L. Systematic review and meta-analysis of clinical efficacy of drug therapy for acute myelogenous leukemia. Ann Palliat Med 2021;10(7):7884-7893. doi: 10.21037/apm-21-1390 pharmacology and use as a surgical aid in ophthalmology, and its therapeutic potential in joint disease and wound healing. Drugs 1994;47:536-66.

13. Philipp-Dormston WG, Bergfeld D, Sommer BM, et al. Consensus statement on prevention and management of adverse effects following rejuvenation procedures with hyaluronic acid-based fillers. J Eur Acad Dermatol Venereol 2017;31:1088-95.

14. Isobe M, Konuma T, Kato S, et al. Development of PreEngraftment Syndrome, but Not Acute Graft-versus-Host Disease, Reduces Relapse Rate of Acute Myelogenous Leukemia after Single Cord Blood Transplantation. Biol Blood Marrow Transplant 2019;25:1187-96.

15. Essendoubi M, Gobinet C, Reynaud R, et al. Human skin penetration of hyaluronic acid of different molecular weights as probed by Raman spectroscopy. Skin Res Technol 2016;22:55-62.

16. Tsepilov RN, Beloded AV. Hyaluronic Acid--an "Old" Molecule with "New" Functions: Biosynthesis and Depolymerization of Hyaluronic Acid in Bacteria and Vertebrate Tissues Including during Carcinogenesis. Biochemistry (Mosc) 2015;80:1093-108.

17. Avasarala SK, Qureshi MR, Waldron M, et al. Initiation of venovenous extracorporeal membrane oxygenation in a patient receiving induction chemotherapy for acute myelogenous leukemia. J Oncol Pharm Pract 2019;25:1491-6.

18. Zuckerman T, Ram R, Akria L, et al. BST-236, a novel cytarabine prodrug for patients with acute leukemia unfit for standard induction: a phase 1/2a study. Blood Adv 2019;3:3740-9.

19. Myles N, Myles H, Clark SR, et al. Use of granulocytecolony stimulating factor to prevent recurrent clozapineinduced neutropenia on drug rechallenge: A systematic review of the literature and clinical recommendations. Aust N Z J Psychiatry 2017;51:980-9.

20. Wright CR, Ward AC, Russell AP. Granulocyte ColonyStimulating Factor and Its Potential Application for Skeletal Muscle Repair and Regeneration. Mediators Inflamm 2017;2017:7517350.

(English Language Editor: L. Huleatt) 